Draft version February 10, 2020

Typeset using $\mathrm{LAT}_{\mathrm{E}} \mathrm{X}$ twocolumn style in AASTeX63

\title{
Spatially-resolved UV diagnostics of AGN feedback: radiation pressure dominates in a prototypical quasar-driven superwind
}

\author{
Jean Somalwar, ${ }^{1}$ Sean D. Johnson, ${ }^{1,2, *}$ Jonathan Stern, ${ }^{3}$ Andy D. Goulding, ${ }^{1}$ Jenny E. Greene, ${ }^{1}$ \\ Nadia L. Zakamska, ${ }^{4}$ Rachael M. Alexandroff, ${ }^{5}$ Hsiao-Wen Chen, ${ }^{6}$ \\ ${ }^{1}$ Department of Astrophysical Sciences, 4 Ivy Lane, Princeton University, Princeton, NJ 08544, USA \\ ${ }^{2}$ The Observatories of the Carnegie Institution for Science, 813 Santa Barbara Street, Pasadena, CA 91101, USA \\ ${ }^{3}$ Department of Physics and Astronomy and CIERA, Northwestern University, Evanston, IL 60208, USA \\ ${ }^{4}$ Department of Physics and Astronomy, Bloomberg Center, Johns Hopkins University, Baltimore MD 21218, USA \\ ${ }^{5}$ Dunlap Institute for Astronomy and Astrophysics, University of Toronto, 50 St. George Street, Toronto, Ontario, Canada M5S $3 H 4$ \\ ${ }^{6}$ Department of Astronomy \& Astrophysics, The University of Chicago, 5640 S Ellis Ave., Chicago, IL 60637, USA
}

(Received; Revised; Accepted)

Submitted to ApJL

\begin{abstract}
Galactic-scale winds driven by active galactic nuclei (AGN) are often invoked to suppress star formation in galaxy evolution models, but the mechanisms driving these outflows are hotly debated. Two key AGN feedback models are (1) radiation pressure accelerating cool gas and (2) a hot outflowing wind entraining the ISM. Highly ionized emission-line diagnostics represent a powerful means of differentiating these scenarios because of their sensitivity to the expected compression of the ISM clouds by the hot wind. Here, we report the first spatially resolved UV emission spectroscopy of a prototypical (radio-quiet) quasar-driven superwind around the obscured quasar SDSS J1356+1026 at $z=0.123$. We observe ratios of $\mathrm{OVI} / \mathrm{CIV}, \mathrm{N}$ V $/ \mathrm{CIV}$, and $\mathrm{CIV} / \mathrm{He}$ II that are remarkably similar for outflowing gas clouds $\lesssim 100 \mathrm{pc}$ and $\approx 10 \mathrm{kpc}$ from the nucleus. Such similarity is expected for clouds with AGN radiation pressure dominated dynamics. Comparing the observed line emission to models of clouds in balance with radiation pressure and/or a hot wind, we rule out the presence of a dynamically important hot wind and constrain the ratio of hot gas pressure to radiation pressure to $P_{\text {hot }} / P_{\text {rad }} \lesssim 0.25$ both at $\lesssim 100 \mathrm{pc}$ and $\approx 10 \mathrm{kpc}$ from the nucleus. Moreover, the predictions of the radiation pressure confined cloud models that best fit observed UV line ratios are consistent with the observed diffuse X-ray spectrum. These results indicate that this AGN superwind is driven by radiation pressure or was driven by a hot wind that has since dissipated despite on-going AGN activity.
\end{abstract}

\section{INTRODUCTION}

Modern models of galaxy evolution often invoke powerful feedback from accreting supermassive black holes $(\mathrm{SMBH})$ in galactic nuclei in order to suppress star formation in massive galaxies (for reviews, see Kormendy \& Ho 2013; Heckman \& Best 2014; Somerville \& Davé 2015). Direct observations of powerful circum-nuclear active galactic nuclei (AGN) driven winds demonstrate that $\mathrm{SMBH}$ feedback is in principle possible, but the physical mechanisms that couple energy and momentum from the nucleus to the interstellar medium (ISM) and surrounding halo gas are fiercely debated (e.g. Morganti 2017; Wylezalek \& Morganti 2018). Two key models for

Corresponding author: Sean D. Johnson

sdj@astro.princeton.edu

* Carnegie-Princeton fellow driving effective, large-scale AGN outflows are (1) direct acceleration of cool gas through radiation pressure (e.g. Murray et al. 2005; Ishibashi \& Fabian 2015; Debuhr et al. 2011; Thompson et al. 2015) and (2) entrainment of the ISM in a hot outflowing wind generated by fast shocks near the nucleus (e.g. Faucher-Giguère \& Quataert 2012; King \& Pounds 2015). Observations that differentiate between these AGN feedback models are necessary for a more complete understanding of galaxy evolution (e.g. Krumholz et al. 2017).

Over the last decade, surveys of outflowing gas around luminous (radio-quiet) AGN demonstrate that multiphase and kinematically disturbed outflows are nearly ubiquitous both near the nucleus $(<1 \mathrm{kpc}$ e.g. Feruglio et al. 2010; Zakamska \& Greene 2014) and on galactic scales of $\approx 10 \mathrm{kpc}$ (e.g. Greene et al. 2011, 2012; Zubovas \& Nayakshin 2012; Liu et al. 2013a,b, 2014; Harrison et al. 2014; Rupke et al. 2017; Sun et al. 2017; Fischer et al. 2018; Husemann et al. 2019; Jarvis et al. 2019). However, 
more in-depth follow-up observations are needed to make definitive statements about the physical conditions in the ongoing outflows, let alone the physical mechanisms that drove or continue to drive them.

Emission line ratios of highly ionized species can diagnose the physical conditions in quasar outflows. These line ratios are sensitive to the ionization level of the H II gas, which in turn depends on the dominant pressure source applied to the illuminated surface of the clouds (Stern et al. 2016). If quasar radiation pressure is the dominant pressure source then the thermal gas pressure at the ionization front roughly equals the incident radiation pressure, implying an ionization parameter of $U \sim 0.03-0.1$. Moreover, in radiation-pressure-dominated clouds, the H II layer has a characteristic density profile and spectral signature in highly-ionized lines (Stern et al. 2014a,b; Baskin et al. 2014a,b; Bianchi et al. 2019). If another pressure mechanism dominates - such as the hot wind - then the gas will have a higher pressure/density and thus a lower ionization state (e.g. Dopita et al. 2002; Stern et al. 2016). In addition, highly ionized lines can differentiate AGN photoionized gas (Groves et al. 2004) from shocks (Allen et al. 2008). Consequently, spectroscopy of emission lines such as O VI, N V, and C IV in the rest-UV represents a sensitive means of testing AGN feedback mechanisms and constraining the elusive hot wind phase.

Here, we present the first sensitive and spatially resolved UV spectra of a prototypical quasar-driven superwind at low redshift, SDSS J135646.10+102609.0 (SDSS J1356+1026). SDSS J1356+1026 is a radio-quiet, obscured quasar at redshift $z=0.123$ driving a prototypical superwind on galactic scales (Greene et al. 2012). The AGN has an estimated bolometric luminosity of $L_{\mathrm{bol}} \approx 2 \times 10^{46} \mathrm{erg} \mathrm{s}^{-1}$ and a black hole mass of $M_{\bullet} \sim 10^{8} \mathrm{M}_{\odot}$ (Sun et al. 2014). The outflow is characterized by kinematically disturbed ionized gas with doublepeaked velocity structure (full width $\gtrsim 800 \mathrm{~km} \mathrm{~s}^{-1}$ in projection, inconsistent with bound gravitational motion) observed in [O III] emission at $\approx 10 \mathrm{kpc}$ from the nucleus that can be modelled as outflowing shells (see Figure 1; Greene et al. 2012). Soft X-ray emission that is detected at the location of the extended outflow can be explained either by the presence of a hot wind or by photoionized line emission (Greene et al. 2014; Foord et al. 2020).

Throughout, we adopt a flat $\Lambda \mathrm{CDM}$ cosmology with $\Omega_{\mathrm{m}}=0.3, \Omega_{\Lambda}=0.7$, and $H_{0}=70 \mathrm{~km} \mathrm{~s}^{-1} \mathrm{Mpc}^{-1}$.

\section{OBSERVATIONS AND DATA REDUCTION}

\subsection{HST COS Data Reduction and Measurements}

We obtained sensitive, spatially resolved FUV emissionline spectra of SDSS J1356+1026 with the Cosmic Origins Spectrograph (COS; Green et al. 2012) on board the Hubble Space Telescope (HST) both with a nuclear pointing on 2019-06-12 and 2019-06-14 (4 orbits; 10.39 sec of exposure; OBSIDs: LDHV02010, LDHV01010; PI: Johnson, PID: 15280) and an off-nuclear pointing on 2018-05-20
(1 orbit; 2.16 ksec of exposure; OBSID: LDHV03010). The off-nuclear pointing is centered at the location of the extended outflow observed in [O III], $4.8 \operatorname{arcsec}(10.6$ $\mathrm{kpc})$ South and $0.6 \operatorname{arcsec}(1.6 \mathrm{kpc})$ West of the nucleus as shown in the middle panel of Figure 1. The COS G140L grating spectra cover key high ionization lines such as the O VI $\lambda \lambda 1031 / 1037, \mathrm{~N}$ V $\lambda \lambda 1238 / 1242, \mathrm{CIV}$ $\lambda \lambda 1548 / 1550$, and Si IV $\lambda \lambda 1394 / 1403$ doublets as well as He II $\lambda 1640$ and the O IV] multiplet at $\lambda 1400$ (blended with Si IV).

We calibrated the COS spectra using the CALCOS pipeline version 3.3.5. Because CALCOS is optimized for point sources, we enlarged the spectral extraction aperture size to $\approx 49$ pixels $(5.4$ arcsec) from the default. Our chosen extraction aperture includes at least $98 \%$ of the total flux under the O VI, N V, and C IV lines, and the line ratios are robust to aperture size changes at the level of $\pm 10 \%$. We combined the extracted individual exposures for each pointing into exposure time weighted final spectra after masking bad pixels. Figure 1 shows the final nuclear and off-nuclear spectra in the top two panels on the right.

The nuclear and off-nuclear UV spectra from COS shown in Figure 1 exhibit emission in $\mathrm{HI}$ Ly $\beta$, O VI, H I Ly $\alpha$, N V, Si IV+O IV], C IV, and HeII $\lambda 1640$. The observed line ratios in the off-nuclear spectrum are strikingly similar to those in the nuclear spectrum. To measure the strengths of the emission lines and quantify this similarity, we fit the spectral region around each line with a linear continuum model and Gaussian emission components as shown in the zoom-in plots in Figure 1. Some features required multiple Gaussians to achieve a good fit. Due to low signal-to-noise in the Si IV+O IV] emission line for the off-nuclear spectrum, we fixed the width of the fit to that from a single Gaussian fit to the same region in the nuclear spectrum. For the same reason, we fixed the width of the Ly $\beta$ off-nuclear Gaussian to be the same as that of the off-nuclear O VI lines. The line measurements are summarized in Table 1 after Milky Way foreground extinction corrections based on Schlafly \& Finkbeiner (2011) and Fitzpatrick \& Massa (2007). The line flux uncertainties include systematic errors of $10-20 \%$ based on flux measurement variations using different continuum models and non-parametric measurements. The $\approx 1^{\prime \prime}$ spatial extent of the emitting gas in the dispersion direction is expected to produce spectral resolution of $\approx 600-1000 \mathrm{~km} \mathrm{~s}^{-1}$ from 1800 $1100 \AA$, consistent with the observed line and doublet full-width-at-half-maxima (FWHM).

\subsection{HST WFC3 Imaging}

To assess the morphological structure of the ionized gas around SDSS J1356+1026, we constructed an [O II] line-map using available wide and medium band $H S T$ images from WFC3+UVIS in the F438W (PI: Comerford, PID: 12754) and F621M (PI: Greene, PID:13944) filters. The F438W filter includes both continuum and line emis- 

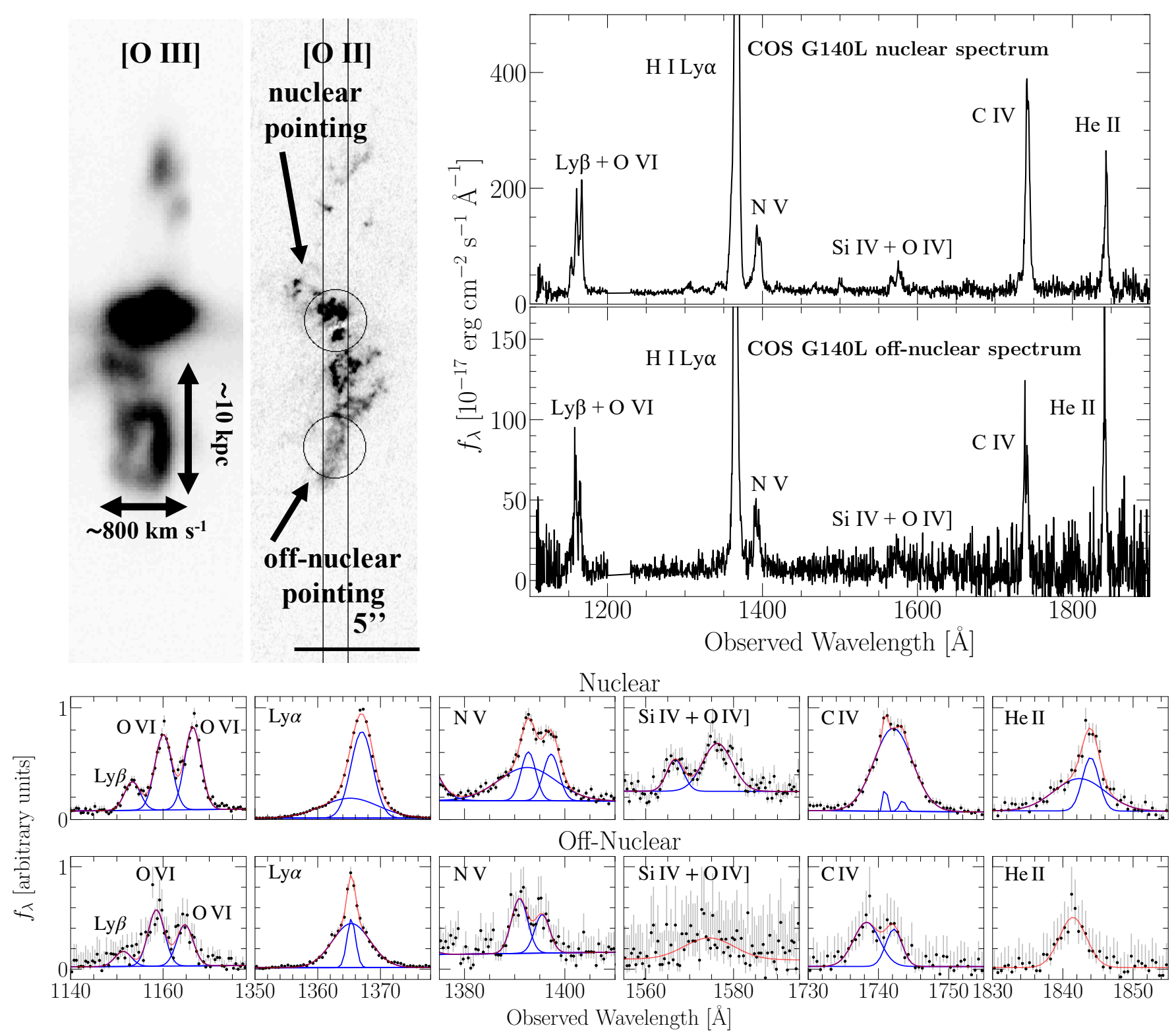

Figure 1. Summary of observations of SDSS J1356+1026, a prototypical quasar-driven superwind at $z=0.123$. The top left panel displays a $2 \mathrm{D}$ longslit spectrum from Magellan centered on the [O III] $\lambda 5007$ line showing extended and kinematically disturbed $\left(\Delta v \approx 800 \mathrm{~km} \mathrm{~s}^{-1}\right)$ [O III] emission $\approx 10 \mathrm{kpc}$ South of the nucleus. The top middle panel displays a high resolution HST image of the [O II] $\lambda 3727$ line emission formed by subtracting the F438W and F621W bands on the same spatial scale as the $2 \mathrm{D}$ spectrum. The emission-line map is dominated by [O II] but may also contain non-negligible contribution from scattered light (e.g. Dempsey \& Zakamska 2018).. The locations of the COS aperture and 1" wide Magellan longslit are overlaid to scale on the HST image for both the nuclear and off-nuclear pointings. The top two panels on the right display the COS spectra from the nuclear and off-nuclear pointings. The bottom panels show zoom-in emission-line spectra for the nuclear (top panels) and the off-nuclear (bottom panels) COS pointings with emission-lines labelled. The best-fit emission model is shown as a red line and individual Gaussians are shown in blue. 
sion, predominantly from the $[\mathrm{O} \mathrm{II}]$ doublet, while the F621M filter is free from strong emission lines. To create the emission line map, we started with the default image reductions from STScI and performed an astrometric alignment and flux scaling prior to image subtraction. To align and scale the images, we identified sixteen serendipitous sources residing in the common field-of-view (FOV). We then estimated the optimal translation, rotation, and flux scaling between the two images by simultaneously minimizing the residuals in subtracted $2^{\prime \prime} \times 2^{\prime \prime}$ cutouts around the serendipitous sources via simulated annealing. The resulting line map is shown in Figure 1.

\subsection{Chandra X-ray Observations}

There are three archival Chandra X-ray observations of SDSS J1356+1026 obtained with the Advanced CCD Imaging Spectrometer (ACIS) in ACIS-S mode taken on dates 2012-03-31 (ObsID:13951; FAINT mode), 201603-29 (ObsID:17047; VFAINT mode), and 2016-05-19 (Obsid:18826; VFAINT mode). Because of the differences in the observing setup and the sensitivity of ACIS over time, we processed each observation separately but consistently using the Chandra software packages in CIAO v4.11 with calibration files from CALDB 4.8.3 applied using chandra_repro. After removing streak events, bad pixels, pixel randomization, cosmic rays, and flares, the final Level-2 events files consist of Good Time Intervals of $19.8,34.8$, and $42.9 \mathrm{ks}$ respectively.

For our analysis of the diffuse X-ray spectrum, we extracted photons and response files using the specextract package from each events file within a region bounded by the $99 \%$ flux contour from the $H S T$ [O II] image. To prevent contamination from the central AGN we masked a central circular region of $2^{\prime \prime}$ radius (90 and $95 \%$ encircled energy fraction at $E=4.5$ and $2 \mathrm{keV}$ respectively). We analyzed the resulting X-ray spectra with XSPEC v12.10 using Cash (1976) statistics and required a minimum of one photon per energy bin.

\section{DISCUSSION}

The observed emission line ratios of $\mathrm{NV} / \mathrm{CIV}$, $\mathrm{C} \mathrm{IV} / \mathrm{He}$ II, and O VI/C IV are shown in Figure 2, and are strikingly similar for the nuclear and off-nuclear observations, despite the factor of $\gtrsim 10^{4}$ difference in ionizing flux. For example, the nuclear $\mathrm{NV}$ to $\mathrm{CIV}$ ratio of $0.40 \pm 0.05$ is consistent with the off-nuclear value of $0.43 \pm 0.08$. This suggests that the density of the emitting clouds has the same $\propto r^{-2}$ radial dependence as the quasar radiation field, as expected if radiation pressure dominates.

To better understand the physical conditions of the emitting clouds, we compare the observed line ratios in Figure 2 to models including radiative shocks (plus photoionized precursor; Allen et al. 2008) and AGN photoionized models calculated with CLOUDY version 17.01 (Ferland et al. 2017). Shocks with velocities greater than $500 \mathrm{~km} \mathrm{~s}^{-1}$ can reproduce the observed emission line ratios of $[\mathrm{O} \mathrm{III}] / \mathrm{H} \beta \approx 10$ at the nucleus and location of the off-nuclear pointing from Greene et al. (2012). However, at these velocities, the shock models significantly over-predict the observed highly ionized line ratios (e.g. $\mathrm{O}$ VI/C IV) as shown in Figure 2. We therefore conclude that AGN photoionization dominates over shock ionization by a large factor in the observed regions of SDSS J1356+1026, though we caution that shocks may still be present in the system (e.g. Zakamska \& Greene 2014).

\subsection{Constant density models}

The observed O VI/C IV ratios can be reproduced by models of AGN photoionized gas clouds with uniform density, $\approx 1-2 \times$ solar metallicity, and ionization parameter of $\log U \approx-0.7$ (Groves et al. 2004), implying $n_{\mathrm{H}} \approx 3 \times 10^{4} \mathrm{~cm}^{-3}$ for $r \lesssim 100 \mathrm{pc}$ and $\approx 2 \mathrm{~cm}^{-3}$ for $r \approx 10 \mathrm{kpc}$ assuming an AGN bolometric luminosity $L_{\text {bol }}=2 \times 10^{46} \mathrm{erg} \mathrm{s}^{-1}$ (Sun et al. 2014) and nominal distances of $100 \mathrm{pc}$ and $10 \mathrm{kpc}$ for the nuclear and offnuclear pointings. Such AGN photoionized gas clouds exhibit an equilibrium temperature of $\approx 10^{4} \mathrm{~K}$ implying gas pressures of $P_{\text {gas }} \approx 10^{8}$ and $\approx 5 \times 10^{4} \mathrm{~K} \mathrm{~cm}^{-3}$ for the nuclear and off-nuclear pointings respectively, $\gtrsim 7 \times$ less than the pressure in the incident radiation, $\widetilde{P}_{\text {rad }} \equiv L /\left(4 \pi r^{2} c\right) \approx 4 \times 10^{9}$ and $4 \times 10^{5} \mathrm{~K} \mathrm{~cm}^{-3}$ respectively. Consequently, neglecting the effect of radiation pressure on the structure of the ionized gas is not justified (e.g. Dopita et al. (2002)).

\subsection{Hydrostatic models including AGN radiation pressure}

To account for the effects of radiation pressure, we employ hydrostatic models of ionized clouds, in which the pressure at the illuminated surface is set by the thermal pressure of the ambient hot gas, $P_{\text {hot }}$, while the momentum transferred to the gas via the absorption of radiation is balanced by a thermal pressure gradient within the ionized cloud. Such models are sometimes referred to as "constant total pressure" models, since the sum of the thermal gas pressure and the remaining pressure in the absorbed radiation is constant throughout the slab. ${ }^{1}$ Confinement on the shielded side is assumed to be provided by the neutral/molecular gas beyond the ionization front. As discussed in Dopita et al. (2002) and Stern et al. (2016), the structure of the ionized cloud depends qualitatively on whether hot gas or radiation is the dominant pressure source. If $P_{\text {hot }} \gg P_{\text {rad }}$ then radiation pressure is negligible and the gas pressure is roughly constant throughout the cloud with $P_{\text {gas }} \approx P_{\text {hot }}$, resulting in a roughly uniform density ionized layer. In contrast if $P_{\text {rad }} \gg P_{\text {hot }}$ then the gas pressure increases

\footnotetext{
1 These models are calculated in CLOUDY using the "constant pressure" option, though they should not be confused with constant gas pressure models in which $P_{\text {gas }}$ is held constant.
} 
Table 1. Summary of observed UV emission line properties

\begin{tabular}{|c|c|c|c|c|c|}
\hline & Line & Flux $\left[10^{-15} \mathrm{erg} \mathrm{cm}^{-2} \mathrm{~s}^{-1}\right]$ & Counts & Centroid $[\AA]$ & FWHM $\left[\mathrm{km} \mathrm{s}^{-1}\right]$ \\
\hline \multirow{7}{*}{ Nuclear } & $\operatorname{Ly} \beta$ & $2.5_{-0.3}^{+0.3}$ & 770 & 1153 & 1000 \\
\hline & O VI 1031.92/1037.61 & $15_{-1}^{+1}$ & 4480 & 1160 & 2700 \\
\hline & H I Ly $\alpha$ & $100_{-10}^{+10}$ & 57281 & 1367 & 1000 \\
\hline & N V 1238.82/1242.80 & $11_{-1}^{+1}$ & 5484 & 1392 & 1900 \\
\hline & Si IV 1393.75/1402.77 + O IV] 1400 & $3.2_{-0.3}^{+0.3}$ & 1030 & 1573 & 2900 \\
\hline & C IV $1548.19 / 1550.77$ & $28_{-3}^{+3}$ & 3347 & 1742 & 1000 \\
\hline & He II 1640.40 & $12.1_{-1}^{+1}$ & 1000 & 1842 & 600 \\
\hline \multirow{7}{*}{ Off-Nuclear } & $\operatorname{Ly} \beta$ & $0.8_{-0.2}^{+0.2}$ & 49 & 1151 & 1100 \\
\hline & O VI 1031.92/1037.61 & $4.9_{-0.6}^{+0.6}$ & 310 & 1161 & 2500 \\
\hline & H I Ly $\alpha$ & $23_{-2}^{+2}$ & 2777 & 1365 & 600 \\
\hline & N V $1238.82 / 1242.80$ & $2.3_{-0.3}^{+0.3}$ & 247 & 1391 & 1600 \\
\hline & Si IV 1393.75/1402.77 + O IV] 1400 & $1.5_{-0.4}^{+0.4}$ & 76 & 1574 & 2500 \\
\hline & C IV $1548.19 / 1550.77$ & $5.3_{-0.7}^{+0.7}$ & 138 & 1739 & 1300 \\
\hline & He II 1640.40 & $5.7_{-0.9}^{+0.9}$ & 101 & 1841 & 700 \\
\hline
\end{tabular}

significantly with depth into the cloud, from $P_{\text {gas }}=$ $P_{\text {hot }}$ at the illuminated surface to $P_{\text {gas }} \approx P_{\text {rad }}$ near the ionization front (see fig. 1 in Stern et al. (2016)). In this case the cloud is Radiation Pressure Confined (RPC), and density and hence ionization parameter are a function of depth so that highly ionized lines arise primarily from outer layers while lower ionization lines arise from deeper layers closer to the ionization front. In RPC clouds, the predicted line ratios are independent of $P_{\text {hot }}$ and exhibit unique spectral signatures. Consequently, highly ionized FUV emission line observations of AGN outflows can serve as effective barometers that enable inferences into whether radiation pressure or a hot wind determine the dynamics of the cool-warm component of AGN driven outflows.

We used CLOUdy to calculate the structure of hydrostatic HII regions photoionized by AGN over a wide range of relative pressures $\left(0.01<P_{\text {hot }} / P_{\text {rad }}<10\right)$. Other model parameters include the dust content of the gas, gas metallicity, the ionizing spectral slope $\alpha$ $\left(L_{\nu} \propto \nu^{-\alpha}\right)$ between 1 Rydberg and $2 \mathrm{keV}$, and the distance to the unresolved nucleus pointing. For the purposes of this letter, these are nuisance parameters. We ran a grid of models with $\alpha=1.6-2$, metallicity in the range $1-2 \mathrm{Z}_{\odot}$, and Milky Way ISM abundances and dust content/depletion (Draine 2011). Because dust may be destroyed in the AGN outflow, we also consider dustfree models which are discussed in the Appendix. Our conclusions are robust to a wide range in choice of these parameters. We assume an AGN bolometric luminosity of $L_{\mathrm{bol}}=2 \times 10^{46} \mathrm{erg} \mathrm{s}^{-1}$, and distances of $10-100 \mathrm{pc}$ for the nuclear pointing and a distance of $10 \mathrm{kpc}$ for the off-nuclear pointing. The predicted line ratios from the models are shown in Figure 2. Dots connected by thick lines denote the model predictions for different $P_{\text {hot }} / P_{\text {rad }}$, for an assumed $\alpha=1.6,1.5 \mathrm{Z}_{\odot}$, and a distance of 100 pc $(10 \mathrm{kpc})$ for the nuclear (off-nuclear) pointing. Thin lines denote predicted line ratios for other choices of these three parameters. Because the cloud models are in the optically thick limit the size and total columns of the emitting regions are not not free parameters. Nevertheless, we ensured that the emitting cloud sizes are smaller than the COS aperture and the corresponding total hydrogen columns range from $N(\mathrm{H})=10^{20}-10^{21.7}$ $\mathrm{cm}^{-2}$.

As $P_{\text {hot }} / P_{\text {rad }} \rightarrow 0$, the predicted line ratios approach asymptotic values, as expected in the radiation pressure dominated limit in which the line ratios are independent of $P_{\text {hot }}$ (Dopita et al. 2002; Stern et al. 2014b). In the dusty models shown in Figure 2, the uncertainty in the predicted line ratios given our assumed range of metallicity, spectral slope and cloud distance is merely $\lesssim 0.1$ dex. All observed line ratios are within $\approx 0.1$ dex of the predictions for radiation pressure dominated clouds for both the nuclear pointing and the off-nuclear pointing. The hot gas pressure dominated models with $P_{\text {hot }}>P_{\text {rad }}$ under-predict $\mathrm{O}$ VI/C IV by an order-of-magnitude and underpredict the observed $\mathrm{NV} / \mathrm{C} \mathrm{IV}$ by a factor of $\approx 3$. The observed line ratios thus strongly disfavor the hot gas pressure dominated models.

\subsection{Any hot wind is currently dynamically unimportant}

The observed N V/C IV, C IV/HeII, and O VI/C IV line ratios for both the nuclear and off-nuclear pointings are consistent with the hydrostatic model predictions in the radiation pressure dominated regime $\left(P_{\text {hot }}<P_{\text {rad }}\right)$ as shown in Figure 2. To quantify the limit on the presence 


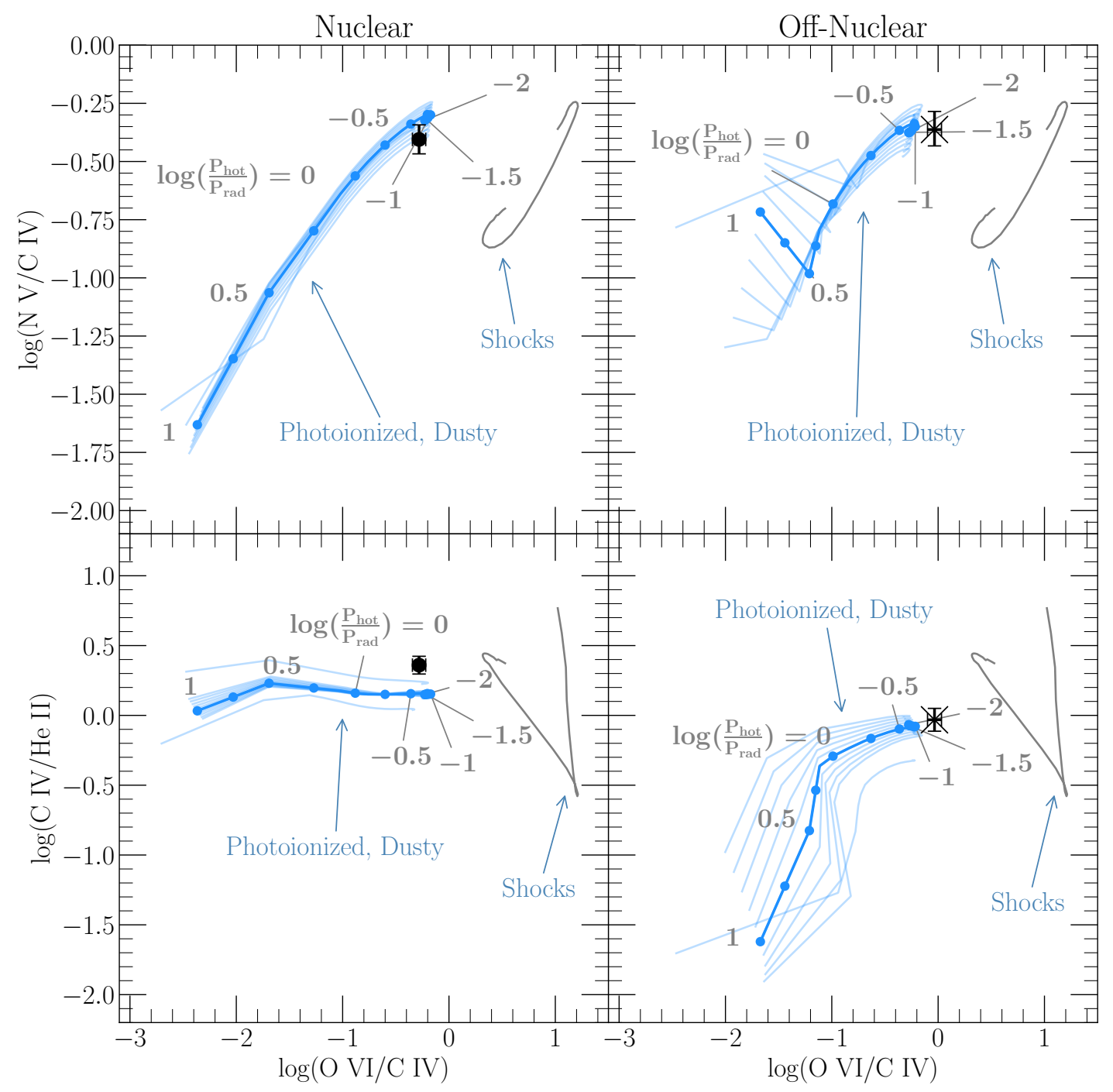

Figure 2. Measured NV/C IV (top) and CIV/He II (bottom) versus O VI/C IV emission line ratios for the nuclear and off-nuclear pointings compared to predictions from shock models and AGN photoionized hydrostatic models. Shock models with shock velocities $>200 \mathrm{~km} \mathrm{~s}^{-1}$ (Allen et al. 2008) are shown in grey and are inconsistent with the observed line ratios. Shock models with lower velocities cannot reproduce the optical line ratios observed in Greene et al. (2012). Hydrostatic AGN photoionization models (blue) include dust grains. The models span $P_{\text {hot }} / P_{\text {rad }}=0.01-10$ in steps of 0.25 dex as marked. Different lines mark different assumptions on the metallicity, spectral slope, and distance of the clouds as described in Section 3.2 . The predicted line ratios approach asymptotic values as $P_{\text {hot }} / P_{\text {rad }} \rightarrow 0$, corresponding to the radiation pressure dominated limit. The observed ratios are most consistent with gas clouds in the radiation pressure dominated limit, ruling out the current presence of a dynamically important hot wind.

of a hot wind component from the UV spectra, Figure 3 displays the observed nuclear and off-nuclear O VI/C IV ratios compared to hydrostatic photoionization model predictions as a function of $P_{\text {hot }} / P_{\text {rad }}$. The thickness of the colored lines denotes the uncertainty in the prediction due to the uncertainty in the nuisance parameters mentioned in the previous section. The observed line ratios for the nucleus fall within the uncertainty range for the dusty model while the off-nuclear ratios fall between the dusty and dust-free models ${ }^{2}$. In both cases, the observed line ratios require $P_{\text {hot }} \lesssim 0.25 P_{\text {rad }}$. We therefore conclude that the outflowing, UV emitting clouds on narrow-line region scales of $\lesssim 100 \mathrm{pc}$ and on galactic scales of $\approx 10 \mathrm{kpc}$ are not currently entrained in a dynam-

2

This suggests intermediate dust content which we will explore in future work. 

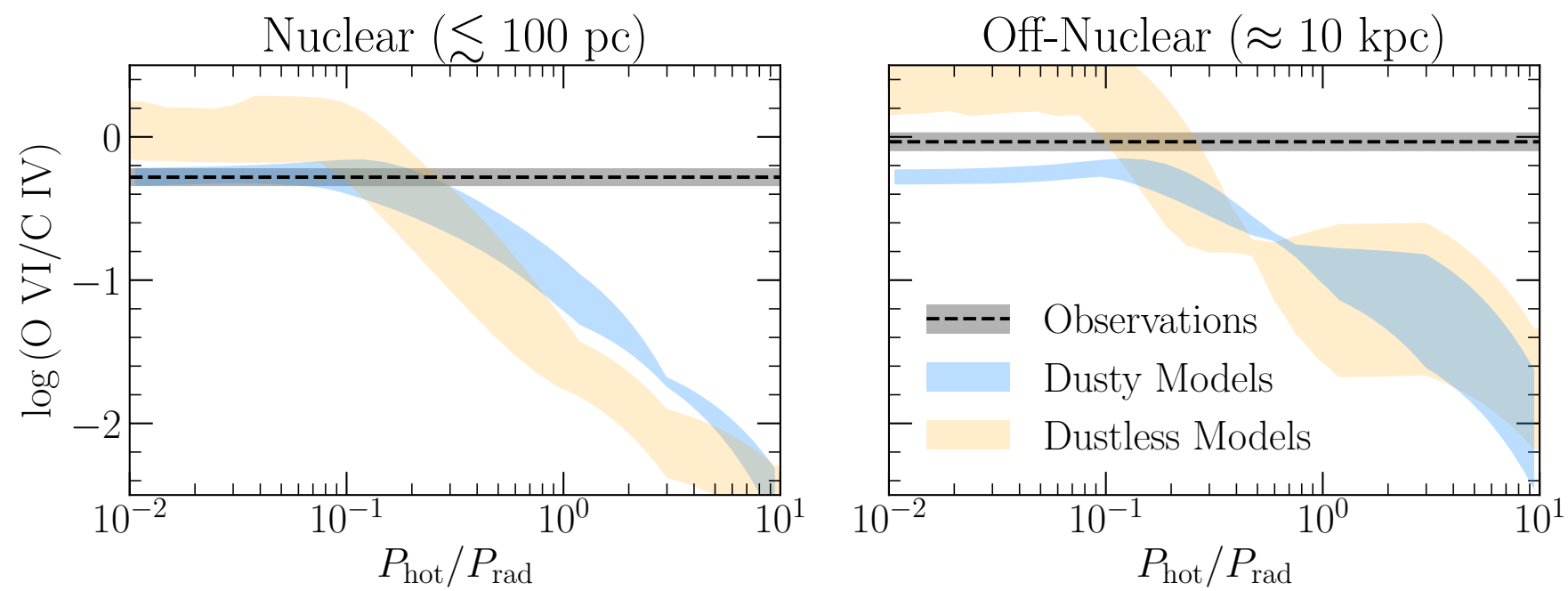

Figure 3. Predicted O VI/C IV emission line ratios as a function $P_{\text {hot }} / P_{\text {rad }}$ for the dusty (dustless) hydrostatic AGN photoionized models in blue (orange) with thickness denoting the predicted range including model uncertainty discussed in the text. The observed line ratios are shown as black dashed lines with grey regions denoting uncertainties. The observed line ratios for both pointings require $P_{\text {hot }} / P_{\text {rad }} \lesssim 0.25$, ruling out the presence of a dynamically important hot wind.
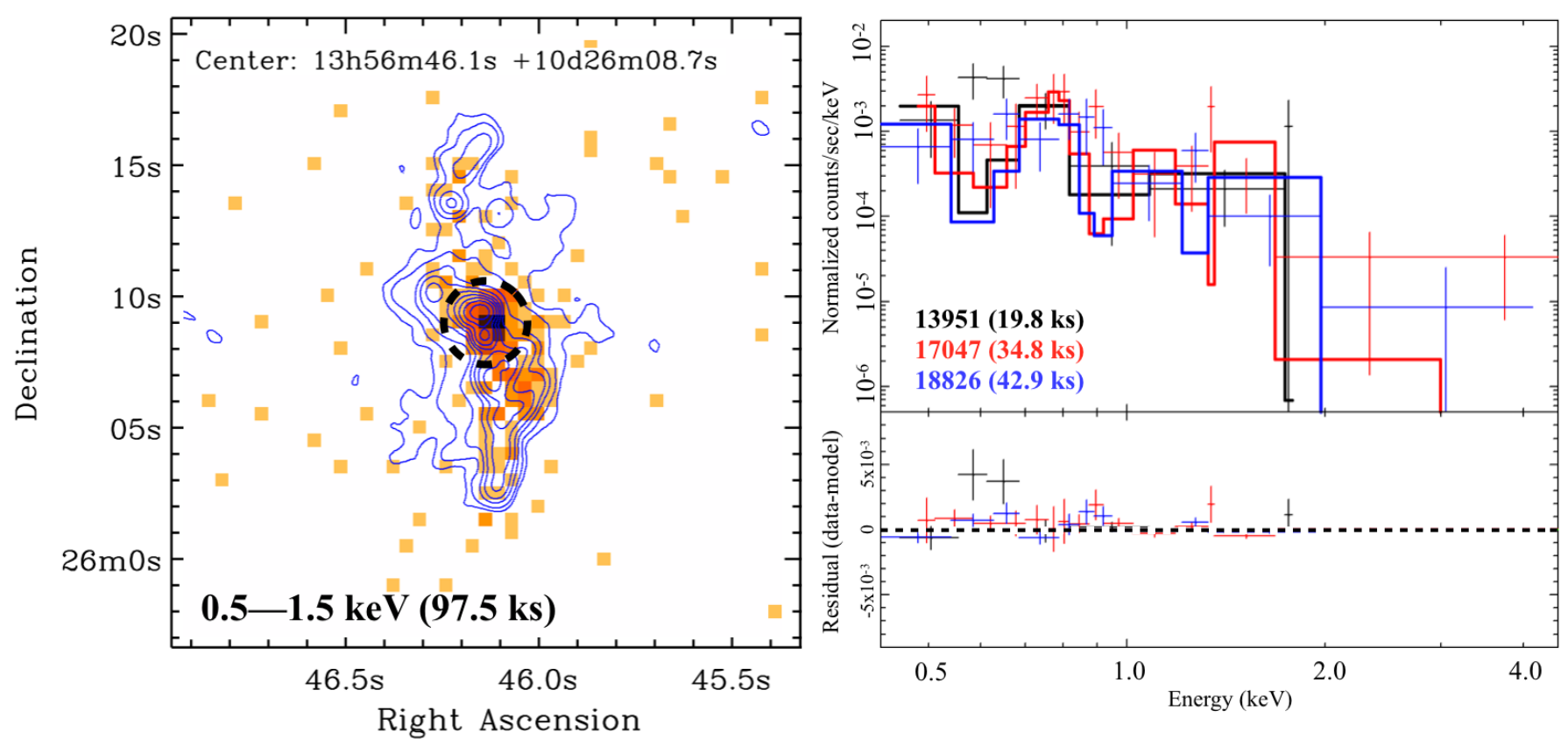

Figure 4. Left: Soft band (0.5-1.5 keV) Chandra X-ray image produced from all three Chandra programs. Contours of emission line regions from HST imaging are overlaid in blue, the outermost contour represents $99 \%$ of the total line flux, and is used to extract X-ray photons for our spectral analysis shown on the right. The circle marks the region masked in the spectral extraction to prevent contamination from the nucleus. Right: X-ray spectra in counts/sec/keV of the diffuse X-ray component extracted from the line emitting region. X-ray data from all three Chandra ObsIDs are shown simultaneously in different colors with a single CLOUDY photoionization model (solid line). Residuals between the X-ray data and the models are shown on the bottom. 
ically important hot wind. Using the estimated radiation pressure at the fiducial distances, we place limits on the current pressure from any hot wind of $P_{\text {hot }}<10^{9}$ and $10^{5} \mathrm{~K} \mathrm{~cm}^{-3}$ for the nuclear and off-nuclear pointings, respectively.

\subsection{The extended $X$-ray emission is consistent with photoionized line emission}

Chandra observations of SDSS J1356+1026 show that the extended outflow of SDSS J1356+1026 emits soft X-rays which can be explained by shocks induced by a hot wind (e.g. Choi et al. 2014; Nims et al. 2015) or by AGN photoionized line emission (e.g. Sambruna et al. 2001). The diffuse, extended X-ray emission from SDSS J1356+1026 is coincident with [O II] emission (see Figure 4), consistent with either scenario. This diffuse component is characterized by low-energy X-ray emission with $E \lesssim 2 \mathrm{keV}$. At harder energies, there are only $7 \mathrm{X}$ ray photons with $E \sim 2-7 \mathrm{keV}$ within the [O II] bounded region, fully consistent with the $6.5 \pm 0.9$ counts expected from the X-ray background in that area in the three combined Chandra exposures.

To test the two scenarios for the origins of the diffuse X-ray emission, we constructed a CLOUDY model of diffuse X-ray emission produced by AGN photoionized gas assuming the $\mathrm{X}$-ray emitting layer is dustless since grains will be destroyed by sputtering in X-ray emitting layers, even if the lower-ionization layers which produce the UV emission are dusty (Stern et al. 2014b). This Cloudy model is consistent with the observed X-ray spectra with a Cash statistic of 109.4 for 85 degrees of freedom (Right panel in Fig. 4) with few significant residuals (lower panel). The diffuse X-ray emission may also be a consequence of shock-heating in a thermally hot plasma. Hence, we also fit the X-ray spectra with an APEC model in XSPEC and find that it is equally consistent with a low best-fit metallicity of $<2 \%$ solar, temperature of $T_{\mathrm{X}} \sim 0.3 \mathrm{keV}$ plasma, and no internal absorption. However, given the consistency between the observed soft X-ray spectrum and the model expectations from the radiation pressure dominated cloud emission observed in the UV, we suggest that the diffuse X-ray emission can be fully explained by photoionized line emission. Conclusively differentiating between the two $\mathrm{X}$-ray scenarios will require future X-ray observatories such as Lynx (Gaskin et al. 2018).

\section{SUMMARY AND CONCLUSIONS}

To gain insights into the physical drivers of AGN feedback on galactic scales, we performed spatially resolved UV emission spectroscopy of a prototypical quasar-driven superwind at low- $z$. Despite the large expected difference in ionizing flux, the observed highly ionized UV line ratios on $\approx 10 \mathrm{kpc}$ scales are similar to those seen near the nucleus $(\lesssim 100 \mathrm{pc})$. This similarity is expected if radiation pressure dominates at the illuminated surface of the line-emitting clouds. Indeed, models of clouds confined by radiation pressure from the AGN self-consistently reproduce the observed UV line ratios as well as the spectral and morphological properties of observed diffuse X-ray emission.

Based on the observed highly ionized UV emission ratios, we rule out the presence of a dynamically important hot wind phase and place an upper limit on the pressure a hot wind may impart to the UV emitting clouds at $\lesssim 10 \mathrm{kpc}$. This upper limit is an order-of-magnitude lower than recent estimates based on tentative detections $(3-4 \sigma)$ of the Sunyaev-Zel'dovich effect around quasars at $z \sim 2-3$ (Hall et al. 2019; Lacy et al. 2019). This tension can be reconciled if AGN feedback varies significantly from object-to-object (e.g. due to luminosity or redshift), if the hot wind is no longer co-spatial with the UV emitting clouds, or if the hot wind has expanded adiabatically and is no longer a dominant pressure source at this stage in the evolution of SDSS J1356+1026. While a hot wind component of the outflow may therefore still exist, the observed highly ionized emission-line ratios indicate that the combined gas pressure and ram pressure from any hot gas are subdominant to the radiation pressure and hence do not confine or provide on-going acceleration to the outflowing, UV emitting clouds. The observed AGN outflow is therefore most likely the result of radiation pressure or a hot wind that accelerated the gas at earlier epochs and has since vented or cooled despite on-going AGN activity, placing novel and stringent constraints on AGN feedback models.

\section{ACKNOWLEDGMENTS}

We are grateful to T. Heckman and E. Schneider for fruitful discussions that influenced this project. We thank the anonymous referee for a constructive and thorough review which strengthened the paper. S. Johnson acknowledges support from NASA through Hubble Fellowship grant (HST-HF2-51375.001-A) awarded by the Space Telescope Science Institute, which is operated by the Association of Universities for Research in Astronomy, Inc., for NASA, under contract NAS5-26555. Based on observations made with the NASA/ESA Hubble Space Telescope through programs GO-12754, GO-13944, and GO-15280 and retrieved from the HST data archive at the Space Telescope Science Institute. Support for this work was provided by NASA through the grant associated with HST-GO-15280. NLZ is supported in part by HST-AR-14592. This paper includes data gathered with the 6.5 meter Magellan Telescopes located at Las Campanas Observatory, Chile. The scientific results reported in this article are based in part on observations made by the Chandra X-ray Observatory. 


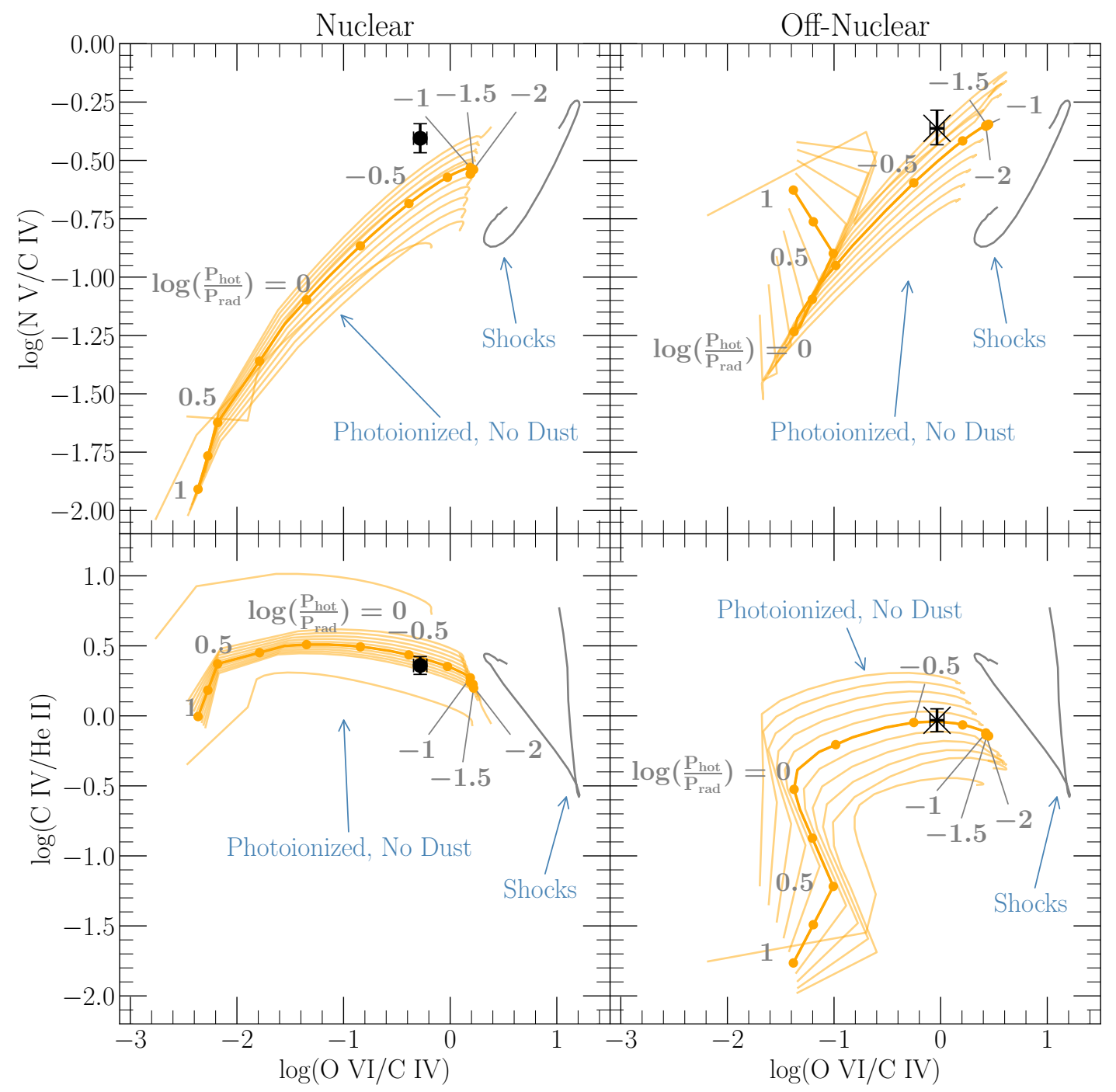

Figure A1. Measured NV/CIV (top) and CIV/HeII (bottom) versus O VI/C IV emission line ratios for the nuclear and off-nuclear pointings compared to predictions from shock models and AGN photoionized hydrostatic models, in the same format as Figure 2. Hydrostatic AGN photoionization models (orange) are dustless. Like the dusty models, the dustless models are most consistent with the observed ratios in the radiation pressure dominated limit, ruling out the current presence of a dynamically important hot wind.

\section{APPENDIX}

\section{A. DUSTLESS MODELS}

Dust content of clouds in RPC can change emission-line ratios not only through extinction and gas-phase depletion, but also by altering the thermodynamic properties of the clouds because the dust absorbs radiation pressure. To ensure that our conclusions are robust to dust content of the clouds we ran model grids as described in Section 3.2 but with no dust and solar relative abundances (Asplund et al. 2009). The resulting line ratio predictions are compared to the observed ones in in Figure A1. Like with the dusty models, the dustless models are most consistent with the observed ratios in the radiation pressure dominated limit, ruling out the current presence of a dynamically important hot wind. 


\section{REFERENCES}

Allen, M. G., Groves, B. A., Dopita, M. A., Sutherland, R. S., \& Kewley, L. J. 2008, ApJS, 178, 20, doi: $10.1086 / 589652$

Asplund, M., Grevesse, N., Sauval, A. J., \& Scott, P. 2009, ARA\&A, 47, 481, doi: 10.1146/annurev.astro.46.060407.145222

Baskin, A., Laor, A., \& Stern, J. 2014a, MNRAS, 438, 604, doi: $10.1093 / \mathrm{mnras} / \mathrm{stt} 2230$

—. 2014b, MNRAS, 445, 3025, doi: 10.1093/mnras/stu1732

Bianchi, S., Guainazzi, M., Laor, A., Stern, J., \& Behar, E. 2019, MNRAS, 485, 416, doi: 10.1093/mnras/stz430

Cash, W. 1976, A\&A, 52, 307

Choi, E., Naab, T., Ostriker, J. P., Johansson, P. H., \& Moster, B. P. 2014, MNRAS, 442, 440, doi: $10.1093 / \mathrm{mnras} / \mathrm{stu} 874$

Debuhr, J., Quataert, E., \& Ma, C.-P. 2011, MNRAS, 412, 1341, doi: 10.1111/j.1365-2966.2010.17992.x

Dempsey, R., \& Zakamska, N. L. 2018, MNRAS, 477, 4615, doi: 10.1093/mnras/sty941

Dopita, M. A., Groves, B. A., Sutherland, R. S., Binette, L., \& Cecil, G. 2002, ApJ, 572, 753, doi: 10.1086/340429

Draine, B. T. 2011, Physics of the Interstellar and Intergalactic Medium

Faucher-Giguère, C.-A., \& Quataert, E. 2012, MNRAS, 425, 605, doi: 10.1111/j.1365-2966.2012.21512.x

Ferland, G. J., Chatzikos, M., Guzmán, F., et al. 2017, RMxAA, 53, 385. https://arxiv.org/abs/1705.10877

Feruglio, C., Maiolino, R., Piconcelli, E., et al. 2010, A\&A, 518, L155, doi: 10.1051/0004-6361/201015164

Fischer, T. C., Kraemer, S. B., Schmitt, H. R., et al. 2018, ApJ, 856, 102, doi: 10.3847/1538-4357/aab03e

Fitzpatrick, E. L., \& Massa, D. 2007, ApJ, 663, 320, doi: $10.1086 / 518158$

Foord, A., Gültekin, K., Nevin, R., et al. 2020, arXiv e-prints, arXiv:2002.01033.

https://arxiv.org/abs/2002.01033

Gaskin, J. A., Dominguez, A., Gelmis, K., et al. 2018, in Society of Photo-Optical Instrumentation Engineers (SPIE) Conference Series, Vol. 10699, Proc. SPIE, 106990N, doi: 10.1117/12.2314149

Green, J. C., Froning, C. S., Osterman, S., et al. 2012, ApJ, 744, 60, doi: 10.1088/0004-637X/744/1/60

Greene, J. E., Pooley, D., Zakamska, N. L., Comerford, J. M., \& Sun, A.-L. 2014, ApJ, 788, 54, doi: 10.1088/0004-637X/788/1/54

Greene, J. E., Zakamska, N. L., Ho, L. C., \& Barth, A. J. 2011, ApJ, 732, 9, doi: 10.1088/0004-637X/732/1/9

Greene, J. E., Zakamska, N. L., \& Smith, P. S. 2012, ApJ, 746, 86, doi: 10.1088/0004-637X/746/1/86
Groves, B. A., Dopita, M. A., \& Sutherland, R. S. 2004, ApJS, 153, 75, doi: 10.1086/421114

Hall, K. R., Zakamska, N. L., Addison, G. E., et al. 2019, MNRAS, 490, 2315, doi: 10.1093/mnras/stz2751

Harrison, C. M., Alexander, D. M., Mullaney, J. R., \& Swinbank, A. M. 2014, Monthly Notices of the Royal Astronomical Society, 441, 3306, doi: $10.1093 / \mathrm{mnras} / \mathrm{stu} 515$

Heckman, T. M., \& Best, P. N. 2014, ARA\&A, 52, 589, doi: 10.1146/annurev-astro-081913-035722

Husemann, B., Scharwächter, J., Davis, T. A., et al. 2019, A\&A, 627, A53, doi: 10.1051/0004-6361/201935283

Ishibashi, W., \& Fabian, A. C. 2015, MNRAS, 451, 93, doi: $10.1093 / \mathrm{mnras} / \mathrm{stv} 944$

Jarvis, M. E., Harrison, C. M., Thomson, A. P., et al. 2019, MNRAS, 485, 2710, doi: 10.1093/mnras/stz556

King, A., \& Pounds, K. 2015, ARA\&A, 53, 115, doi: 10.1146/annurev-astro-082214-122316

Kormendy, J., \& Ho, L. C. 2013, ARA\&A, 51, 511, doi: 10.1146/annurev-astro-082708-101811

Krumholz, M. R., Thompson, T. A., Ostriker, E. C., \& Martin, C. L. 2017, MNRAS, 471, 4061, doi: $10.1093 / \mathrm{mnras} / \mathrm{stx} 1882$

Lacy, M., Mason, B., Sarazin, C., et al. 2019, MNRAS, 483, L22, doi: 10.1093/mnrasl/sly215

Liu, G., Zakamska, N. L., \& Greene, J. E. 2014, MNRAS, 442, 1303, doi: 10.1093/mnras/stu974

Liu, G., Zakamska, N. L., Greene, J. E., Nesvadba, N. P. H., \& Liu, X. 2013a, MNRAS, 436, 2576, doi: $10.1093 / \mathrm{mnras} / \mathrm{stt} 1755$

—. 2013b, MNRAS, 430, 2327, doi: 10.1093/mnras/stt051

Morganti, R. 2017, Frontiers in Astronomy and Space Sciences, 4, 42, doi: 10.3389/fspas.2017.00042

Murray, N., Quataert, E., \& Thompson, T. A. 2005, ApJ, 618, 569, doi: 10.1086/426067

Nims, J., Quataert, E., \& Faucher-Giguère, C.-A. 2015, MNRAS, 447, 3612, doi: 10.1093/mnras/stu2648

Rupke, D. S. N., Gültekin, K., \& Veilleux, S. 2017, ApJ, 850, 40, doi: 10.3847/1538-4357/aa94d1

Sambruna, R. M., Netzer, H., Kaspi, S., et al. 2001, ApJL, 546, L13, doi: 10.1086/318068

Schlafly, E. F., \& Finkbeiner, D. P. 2011, ApJ, 737, 103, doi: 10.1088/0004-637X/737/2/103

Somerville, R. S., \& Davé, R. 2015, ARA\&A, 53, 51, doi: 10.1146/annurev-astro-082812-140951

Stern, J., Behar, E., Laor, A., Baskin, A., \& Holczer, T. 2014a, MNRAS, 445, 3011, doi: 10.1093/mnras/stu1960 
Stern, J., Faucher-Giguère, C.-A., Zakamska, N. L., \& Hennawi, J. F. 2016, ApJ, 819, 130, doi: 10.3847/0004-637X/819/2/130

Stern, J., Laor, A., \& Baskin, A. 2014b, MNRAS, 438, 901, doi: 10.1093/mnras/stt1843

Sun, A.-L., Greene, J. E., \& Zakamska, N. L. 2017, ApJ, 835, 222, doi: 10.3847/1538-4357/835/2/222

Sun, A.-L., Greene, J. E., Zakamska, N. L., \& Nesvadba, N. P. H. 2014, ApJ, 790, 160, doi: 10.1088/0004-637X/790/2/160
Thompson, T. A., Fabian, A. C., Quataert, E., \& Murray, N. 2015, MNRAS, 449, 147, doi: 10.1093/mnras/stv246

Wylezalek, D., \& Morganti, R. 2018, Nature Astronomy, 2, 181, doi: 10.1038/s41550-018-0409-0

Zakamska, N. L., \& Greene, J. E. 2014, MNRAS, 442, 784, doi: $10.1093 /$ mnras/stu842

Zubovas, K., \& Nayakshin, S. 2012, MNRAS, 424, 666, doi: $10.1111 /$ j.1365-2966.2012.21250.x 